\title{
Effect of lithium in the DIII-D SOL and plasma-facing surfaces*
}

\author{
G.L. Jackson ${ }^{1}$, C.P. Chrobak ${ }^{1}$, A.G. McLean ${ }^{2}$, R. Maingi ${ }^{3}$, D.K. Mansfield ${ }^{3}$, A.L. Roquemore ${ }^{3}$, \\ P. Diwakar ${ }^{4}$, A. Hassanein ${ }^{4}$, A. Lietz ${ }^{6}$, D.L. Rudakov ${ }^{5}$, T. Sizyuk $^{4}$, \\ and J. Tripathi ${ }^{4}$ \\ ${ }^{1}$ General Atomics, PO Box 85608, San Diego, California 92186-5608, USA \\ ${ }^{2}$ Lawrence Livermore National Laboratory, 700 East Ave, Livermore, California 94550, USA \\ ${ }^{3}$ Princeton Plasma Physics Laboratory, PO Box 451, Princeton, New Jersey 08543-0451, USA \\ ${ }^{4}$ Purdue University, West Lafayette, Indiana 47907, USA \\ ${ }^{5}$ University of California San Diego, 9500 Gilman Dr., La Jolla, California 92093-0417, USA \\ ${ }^{6}$ University of Illinois
}

\begin{abstract}
Lithium has been introduced into the DIII-D tokamak, and migration and retention in graphite have been characterized since no lithium was present in DIII-D initially. A new regime with an enhanced edge electron pedestal and $\mathrm{H}_{98 \mathrm{y} 2} \leq 2$ has been obtained with lithium. Lithium deposition was not uniform, but rather preferentially deposited near the strike points, consistent with previous ${ }^{13} \mathrm{C}$ experiments. Edge visible lithium light (LiI) remained well above the previous background during the entire DIII-D campaign, decaying with a 2600 plasma-second e-fold, but plasma performance was only affected on the discharge with lithium injection. Lithium injection demonstrated the capability of reducing hydrogenic recycling, density, and ELM frequency.

Graphite and silicon samples were exposed to a lithium-injected discharge, using the DiMES system and then removed for ex-situ analysis. The deposited lithium layer remained detectable to a depth up to $1 \mu \mathrm{m}$.
\end{abstract}


Abstract length (150 words): 142 currently

PACS: 52.30.-q, 52.40.Hf, 52.55.Fa

PSI-20 keywords: Plasma Flow, DIII-D, Divertor diagnostic, Spectroscopy, Lithium

*Corresponding author address: General Atomics, 3550 General Atomics Court, San Diego, CA 92121

*Corresponding author E-mail: jackson@fusion.gat.com 


\section{Introduction}

The introduction of lithium as a low $\mathrm{Z}$ wall coating has led to a reduction in hydrogenic recycling and impurity influxes and improved performance in many plasma devices [1-6]. The DIII-D tokamak [7] is uniquely suited to contribute to this research especially in areas of lithium migration, retention in Plasma Facing Components (PFCs) and changes in the plasma-edge dynamics. Lithium wall coatings have been applied to plasma facing surfaces in many devices with a variety of deposition methods, e.g. pellets, powder, evaporation, and surface wetting $[1-4,8,9]$. While lithium has been studied in tokamaks for over 2 decades, an understanding of its behavior, especially in a high temperature plasma environment is still evolving. Although the ultimate goal of introducing lithium into DIII-D is to improve plasma performance and characterize the H-mode edge pedestal, the absence of lithium in the DIII-D graphite tiles at the beginning of these experiments allows a unique opportunity to directly assess and compare the effects of lithium, especially its migration from the injection point to its interaction and retention in PFCs. In DIII-D, nearly the entire plasma- facing surface, except for port openings, is covered with graphite as the PFC material.

In the work reported here, lithium powder, $44 \mu \mathrm{m}$ diameter, is dropped from the top of DIII-D using a piezo-electric shutter and associated hardware; details of the Lithium Dropper (LiDr) have been reported elsewhere [9]. For DIII-D the typical injection velocity entering the torus due to acceleration by gravity is $\approx 6-7 \mathrm{~m} / \mathrm{s}$ and injection rates up to $0.15 \mathrm{~g} / \mathrm{s}$ have been measured in the laboratory. Shown in Fig. 1 is the location of the LiDr and the MHD equilibria for the two types of plasmas used in this work: a lower single null (LSN) discharge, Fig. 1(a), and a double null divertor (DND), Fig. 1(b). Also shown are the viewing geometries of the multi-channel divertor spectrometer (MDS) chords. 
In this paper we will first discuss lithium migration from the injection point to the PFCs. Lithium deposited on these PFCs diffuses into the graphite [10] so it is only partially effective in reducing recycling and improving plasma performance; hence observations of Li retention are presented in Section 3. It may be necessary to remove this lithium from PFCs for future DIII-D experiments, so removal techniques are also investigated. Section 4 discusses results of the effect of lithium on DIII-D plasmas including a new regime with an enhanced edge pedestal and Section 5 presents conclusions.

\section{Migration of Lithium to PFCs}

A key question to be addressed is: how the injected lithium reaches PFCs and where on these surfaces it is deposited? Since the lithium in this work consists of very small particles at low velocity, neutral penetration should be rather shallow and prompt ionization is expected. This was confirmed using Parks' pellet ablation model [11] where ionization occurred outside the separatrix for typical DIII-D pulses. Hence, the dominant flow might be expected from the injector directly to the vicinity of the strike points, as has been reported for ${ }^{13} \mathrm{C}$ trace impurity injection [12].

Lithium migration to the PFCs is observed experimentally with LiI radiation viewed by the MDS. Neutral lithium line radiation $(670.8 \mathrm{~nm})$ occurs near the wall in the far SOL region and the MDS can identify the poloidal locations of this lithium light. The spectrometer is located at $150^{\circ}$ toroidally, well away from the LiDr at $285^{\circ}$. The viewing geometry of the MDS chords is shown in Fig. 1, and the regions of highest intensity marked by thick lines. Thus the flow of ionized lithium is observed dominantly near the strike points. The predicted flow, discussed below, is indicated by arrows in Fig. 1. For the LSN, shown in Fig. 1(a), the highest fraction is observed at the lower inner strike location (chord L2). The inner midplane chord, R0, also 
shows a higher lithium fraction after this injection (with SOL flow predicted on the high field side) when compared to Fig. 1(b) where SOL flow is predicted on the low field side. For the DND case, Fig. 1(b), chord U5 also exhibited high LiI intensity indicating local deposition poloidally near the injector port. The differences in SOL flow are also consistent with the observation (not shown) that the largest observed Zeeman splitting of the LiI line occurs for the case with inferred SOL flow down the inner wall, where the toroidal magnetic field is highest. We note that the direction of SOL flow discussed above is consistent with previous DIII-D ${ }^{13} \mathrm{C}$ experiments [12].

\section{Li Retention in, and Removal from, Graphite}

The observed distribution of LiI radiation discussed in Section 2 persists for many discharges after lithium injection; thus lithium is retained in the graphite tiles that make up the DIII-D PFCs. Although Li retention has been observed in other tokamaks [13] the absence of Li before this work and a precise measurement of injected lithium allowed a careful assessment of lithium retention and also it's presence in ensuing tokamak discharges.

LiI intensity, inferred from MDS has a very slow decay when observed in subsequent plasma pulses, shown in Fig. 2. It is important to note that while lithium is observed in the SOL in subsequent pulses, its effect on the plasma discharge, discussed in the next section, is only observed on the pulse with lithium injection. These data in Fig. 2 are for a specific MDS chord, but similar behaviour is observed for all MDS channels that detected lithium. The thick dashed line in Fig. 2 represents an order of magnitude reduction that requires 6000 plasma-s (2600 plasma-s e-fold decay), or a significant amount of the entire 2013 campaign to approach the pre-injection baseline level (a typical DIII-D discharge is approximately 5 -6 plasma-s). Three bakes to $350^{\circ} \mathrm{C}$ accompanied by a boronization each increased the lithium levels on subsequent 
discharges to approximately the level after injection, implying a reservoir of lithium in the graphite that can diffuse to the surface at higher temperature, even in the presence of a boron film.

To further examine this lithium retention in the DIII-D graphite tiles, a surface sample was inserted into DIII-D, using the Divertor Materials Evaluation System (DiMES) [14]. A silicon wafer with a graphite holder was inserted flush with the graphite floor at the same poloidal position as the LiDr, but displaced by $135^{\circ}$ toroidally. The bulk graphite and sample were at room temperature and the divertor strike point was well away from the sample, which was located approximately at the MDS chord L5 viewing location. The sample was exposed to 2 pulses: a lithium injection pulse [discharge in Fig. 1(b)] and the next pulse without lithium injection. The silicon sample, and sections of the graphite holder were then analysed at the Purdue Center for Materials under Extreme Environment using X-ray electron spectroscopy (XPS), Auger electron spectroscopy (AES), laser induced breakdown spectroscopy (LIBS), and a scanning electron microscope (SEM). XPS measured the near-surface composition $(\approx 1-$ $10 \mathrm{~nm})$ of the silicon sample to be Li (12.6\%), carbon (72.7\%) and oxygen (14.7\%). We note that the sample was exposed to air after plasma exposure, so the oxygen content does not represent the in-situ composition.

LIBS was used to characterize the lithium penetration into the silicon sample. Nine $1 \mathrm{~mm}$ dia. spots were ablated to assure that there were no anomalies in the data; relative standard deviation was $7.1 \%$. The averaged depth profile is shown in Fig. 3. There is a rapid decrease in lithium from the surface, then a slow decay as the sample is further ablated. Simulation with the HEIGHTS package [15] showed that the depth ablated by 20 pulses corresponds to $1000 \mathrm{~nm}$. 
The silicon sample was also cross-sectioned and examined with the SEM. This depth was also measured to be $\approx 1000 \mathrm{~nm}$, consistent with the LIBS measurements.

Further ex-situ analysis was done at the end of the 2013 campaign by removing 25 graphite samples distributed toroidally and poloidally around the torus. The removal method was to scrape small areas in the graphite tiles with a carbide tool (estimated depth $\approx 50-100 \mu \mathrm{m}, \mathrm{A} \approx$ $1 \mathrm{~cm}^{2}$ ) and analyze each residue. The total lithium content was determined using inductively coupled plasma mass spectroscopy. From this analysis we can make the following observations:

- Peaked concentrations are near the injector location, the midplane centerpost, and the lower floor.

- Both toroidal and poloidal asymmetries are observed; poloidal asymmetries are qualitatively consistent with MDS measurements.

- Lithium in the DIII-D torus is estimated to be $0.54 \mathrm{~g}+/-70 \%$.

The estimate of total lithium is approximately twice the measured injected lithium, though within the estimated uncertainty. One reason for the discrepancy in inventory is the limited number of samples available to account for toroidal and poloidal asymmetries. The sample locations emphasized (but were not limited to) known regions of high lithium deposition inferred from the MDS spectrometer.

While lithium conditioning can have positive effects on plasma performance there may also be deleterious effects, especially for diagnostics. For example DIII-D has a lithium beam (LIBEAM) diagnostic to measure the edge current density profile and it is sensitive to background lithium. In addition, many diagnostics measuring light, from infra-red to ultraviolet are absolutely calibrated and window coatings would be problematic. Thus, techniques were 
investigated to remove lithium from the DIII-D graphite tiles, if required in the future. Window coatings can generally be mitigated during a machine opening by physical cleaning. However removal from large area plasma facing surfaces, especially graphite can be more difficult. Hence, graphite samples, taken from the DiMES graphite sample holder exposed to the same two pulses discussed above were analysed ex-situ to determine the most effective removal techniques. The LIBS results are summarized in Table I. As might be expected, physical removal by sanding is the most effective means of removal. However, a simple application of commercial acetic acid (white vinegar) was also effective. These data will determine what techniques might be used if future lithium removal from DIII-D is necessary.

Table I. Normalized Lithium Fraction Using Various Removal Techniques

\begin{tabular}{|l|c|}
\hline Graphite Sample & Normalized Integrated LiI (LIBS) \\
\hline Control & 1 \\
\hline Ethanol Wipe & 0.839 \\
\hline Ethanol + Water & 0.713 \\
\hline Sanded + Alcohol & 0.39 \\
\hline Vinegar Wipe & 0.180 \\
\hline Sanded Twice & 0.026 \\
\hline Sanded 4 Times & 0.013 \\
\hline
\end{tabular}

\section{Effect of Lithium on DIII-D Plasma Discharges}

In proof-of-principle experiments in 2013, $0.26 \mathrm{~g}$ of lithium was injected in 5 discharges. Although this was a rather modest amount compared to other tokamaks, it allowed for some initial observations in DIII-D plasmas. A comparison of 3 discharges is shown in Fig. 4: before-lithium, with lithium, and post-lithium. Comparing the phase with similar conditions, $\mathrm{t}>4.1 \mathrm{~s}, \mathrm{D}_{\alpha}$ line intensity is clearly reduced and ELM frequency is lower on the lithium discharge. In addition, radiated power is similar and density is modestly lower. Charge exchange recombination of fully stripped lithium detected by the EUV survey spectrometer 
(SPRED) indicates that lithium has penetrated the separtatrix. This LiIII data shows both a charge exchange component from near the plasma core, modulated due to the neutral beam duty cycle used for the charge exchange, superimposed on a baseline contribution from edge electron impact excitation and recombination. A more precise measurement of the core lithium using the visible CER profile spectrometer shows an exponential decay and an inferred lithium particle confinement time of $0.41 \mathrm{~s}$ (measured at $\rho_{\mathrm{N}}=0.5,3.6 \leq \mathrm{t} \leq 4.7 \mathrm{~s}$, and assuming no lithium recycling), about twice the energy replacement time of $0.22 \mathrm{~s}$. It is important to note that core lithium was not detected on pulses either before or after lithium injection.

Recent experiments in 2014 have shown that lithium can modify the edge pedestal profile, leading to an significant enhancement in electron pedestal density width, temperature, and pressure, with energy confinement, $\mathrm{H}_{98 \mathrm{y}, 2}$, increasing up to 2.1 during the ELM free period. This increased performance follows a bifurcation in edge pedestal parameters, shown in Fig. 5. In Fig. 5, $\mathrm{n}_{\mathrm{e}, \mathrm{ped}}$, $\mathrm{T}_{\mathrm{e}, \mathrm{ped}}$, and hence $\mathrm{P}_{\mathrm{e}, \mathrm{ped}}$ increased by factors of 1.5, 1.9, and 2.9 respectively during the ELM free period. The ELM free phase was terminated as the discharge reached the peeling-ballooning limit. While the pedestal pressure was higher before the next ELM with lithium, the normalized pedestal pressure (inferred from ELITE calculations) was similar for both pulses in Fig. 5 [16], i.e. the enhanced pedestal allowed higher absolute pedestal pressure, but did not significantly affect the peeling-ballooning boundary. These results were achieved with only a modest amount of lithium, $0.018 \mathrm{~g} / \mathrm{sec}$.

\section{Summary}

Lithium injection has been successfully achieved in the DIII-D tokamak. A new regime with enhanced pedestal $\mathrm{P}_{\mathrm{e}}, \mathrm{n}_{\mathrm{e}}$, and $\mathrm{T}_{\mathrm{e}}$ has been observed with only modest amounts of injected lithium. The reason for this enhanced performance is still being investigated; the working 
hypothesis is that with lithium, the plasma edge remains below the peeling-ballooning limit and hence the ELM-free duration is extended [16].

Since lithium had not been previously used for more than a decade, no lithium background was detected with DIII-D spectrometers (the LiB only injects trace amounts of Li) before the LiDr was used. Hence, a detailed analysis the effect of lithium, especially for retention and migration using the extensive complement of diagnostics on DIII-D can be made. We note that this background does not affect recycling or change plasma performance; only lithium injected on a given shot affects that particular discharge. Hence, routine operation with lithium might require continual injection of lithium. However, to date only $1.2 \mathrm{gm}$ has been injected so long term assessments cannot be made. We note that for NSTX there is a modest cumulative effect from shot-to-shot, although best performance still requires lithium before every discharge [5]. Ex-Situ analysis of samples exposed to lithium, using DiMES, indicated that lithium once deposited, can diffuse or intercalate to depths up to $1000 \mathrm{~nm}$. This is consistent with observations from other work [10,13]. In addition, 25 graphite samples exposed during the entire 2013 campaign showed that lithium deposited in the tiles was very non-uniform, consistent with observations by MDS. Both toroidal and poloidal asymmetries were observed, but the limited number of samples did not allow detailed analysis. Clearly, however, lithium diffuses into the graphite to significant depths, leading to asymmetries that persist for long periods. Increased temperature, i.e. baking, was observed to increase the SOL lithium (and hence possible transport to other locations) and it is possible that this mechanism might lead to more homogeneous distribution over time, but this requires further study.

Analyses of graphite samples exposed to lithium injection were tested for various methods of lithium removal. As expected, physical removal by sanding the graphite surfaces was most 
effective, but surprisingly, the application of acetic acid followed by wiping the surface lowered the lithium in the tiles to 0.18 of its original density. These data, along with the non-uniformity of the lithium deposition can be used to optimize lithium removal from the DIII-D tokamak should this be necessary.

While lithium was only observed inside the LCFS for a single pulse, neutral lithium line radiation was observed on all chords of the MDS visible spectrometer and this effect showed only a slow decay: a factor of 10 reduction in 6000 plasma seconds. While the neutral lithium had no effect on plasma discharges after the initial drop, it did interfere with measurements from the LIBEAM diagnostic, which cannot operate simultaneously with LiDr experiments without modifications.

Future work will focus on detailed experiments to characterize the effect of lithium on the H-mode pedestal (especially understanding the physics of the new pedestal bifurcation regime), recycling, density control, changes in ELM behavior, and improvements in global confinement.

\section{Acknowledgment}

This work was supported in part by the US Department of Energy under DE-FC02-04ER54698, DE-AC52-07NA27344, DE-AC02-09-CH1466 and DE-SC0001961. We are grateful for the lithium dropper hardware supplied by Princeton Plasma Physics Laboratory and to Drs. D.K. Mansfield and A.L. Roquemore for assisting in installation and experimental support. We also acknowledge the work of Prof. A. Hassanein and his research staff at the Center for Materials under Extreme Environment, Purdue University in performing ex-situ analysis of the DIII-D samples. DIII-D data shown in this paper can be obtained in digital format by following the links at https://fusion.gat.com/global/D3D_DMP. 


\section{References}

[1] Mansfield, D.K. et al Phys. Plasmas 3 (1996) 1892

[2] Zuo, G. Z. Plasma Phys. Control. Fusion 54 (2012) 015014

[3] Apicella, M. et al J. Nucl. Mater. 363-365, (2007) 1346

[4] Masjeskii, R. et al Phys. Rev. Lett. 97075002 (2006)

[5] Maingi, R. et al Phys. Rev. Lett. 107145004 (2011)

[6] Bell, M. G. et al Plasma Phys. Controlled Fusion, 51124054 (2009)

[7] Luxon, J. L. Nucl. Fusion 42 614-633

[8] Jackson, G. L. et al J. Nuc. Mater. 241-243(1997) 655.

[9] Mansfield, D. K. et al Fusion. Eng. Design 85 (2010) 890

[10] Itou, N., et al J. Nucl. Mater. 290 281-285 (2001)

[11] Parks, P. B., et al Nuclear Fusion 34417 (1994)

[12] Stangeby, P.C. J. Nucl. Mater. 415 S278 (2011)

[13] Allain, J. P. and Taylor, C. N. Phys. Plasmas 19056126 (2012)

[14] Wong, C. P. C. et al J. Nucl. Mater. 196-198 871 (1992)

[15] Hassanein, A. private communication (2014)

[16] Jackson, G.L., et al, "Enhanced Pedestal Performance in DIII-D Elm-free H-modes with Lithium Injection", Proc. $25^{\text {th }}$ IAEA Fusion Energy Conference, St. Petersburg, Russia (2014) 


\section{Figure Captions}

Fig. 1. MHD Equilibria for two discharges with lithium injection and two different directions of Ip: (a) \#152742, lower single null, and (b) \#153374, double null, with assumed SOL flow shown in red and discussed in Section 2. Viewing geometry of 13 MDS chords are shown and chord with highest lithium intensity in each case is shown as a thick line. The poloidal location of the lithium dropper is also shown.

Fig. 2. Long term trend of neutral LiI emission, measured by MDS, for most of the DIII-D 2013 campaign. Significant events, i.e. lithium injection or boronization and bake, are denoted by vertical lines. Background level is determined from the 2012 campaign.

Fig. 3. Depth profile of lithium using LIBS for a silicon sample exposed to two DIII-D discharges including one with lithium injection (\#153374). Ablation for 20 laser pulses corresponds to a depth of $\approx 1000 \mathrm{~nm}[15]$.

Fig. 4. Effect of lithium for pulse 152742 (red) compared to pulses before (black) and after (blue) injection. For $\mathrm{t}>4.1 \mathrm{~s}$, discharge conditions are the same for all 3 pulses. Lowest $\mathrm{D}_{\alpha}$ intensity is observed after lithium injection, (a) Radiated power, (b) is similar and density, (c) is reduced later in time, $\mathrm{t}>4.6 \mathrm{~s}$. LiIII intensity is also shown, (d). Discharge conditions: lower single null $(\mathrm{LSN}), \mathrm{I}_{\mathrm{p}}=1.2 \mathrm{MA}, \mathrm{B}_{\mathrm{T}}=2.1 \mathrm{~T}, \mathrm{P}_{\mathrm{NB}}(\mathrm{t}>4.1 \mathrm{~s})=2.4 \mathrm{MW}$. Lithium injection occurred from $2.5-3.5 \mathrm{~s}$ during beam heated $\mathrm{H}$-mode.

Fig. 5. Increase in electron pedestal pressure, (a); electron pedestal width, (b); H factor, (c), for a discharge with Li injection, (\#157278) compared to a discharge without Li (\#157263). ELM-free period is shown in (d), with $\mathrm{Li}$, and (e), without $\mathrm{Li}$. Li injection is from $2.0-3.0 \mathrm{~s}$, $0.018 \mathrm{~g} / \mathrm{s}$. Time of bifurcation is shown as a vertical line. Plasma parameters are: $\mathrm{I}_{\mathrm{p}}=1.2 \mathrm{MA}$, $\mathrm{B}_{\mathrm{T}}=2.0, \mathrm{q}_{95}=4.5$. 
P2-108 


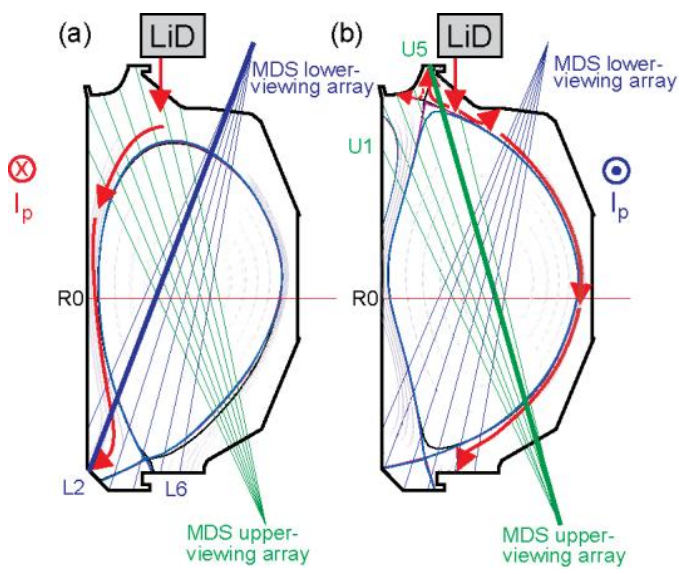

Fig. 1 


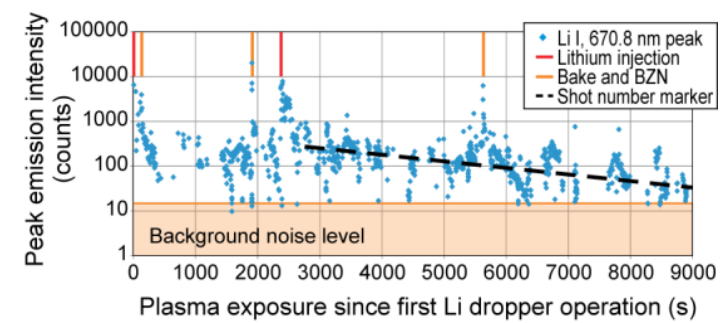

Fig. 2 


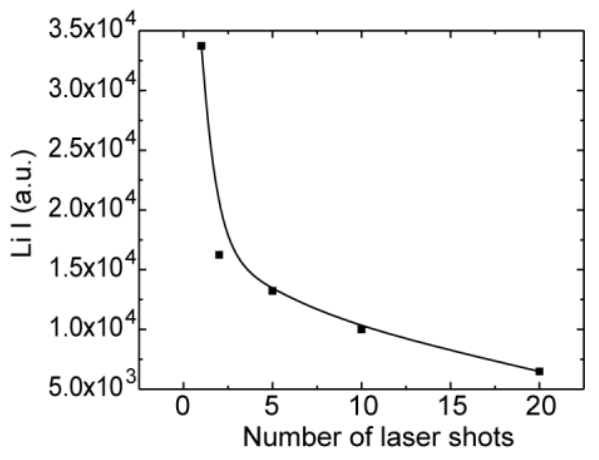

Fig. 3 


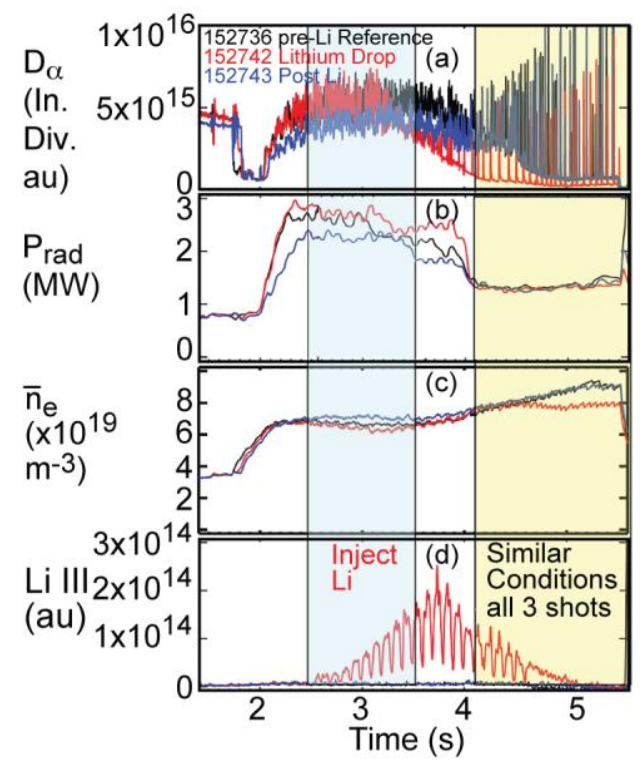

Fig. 4 


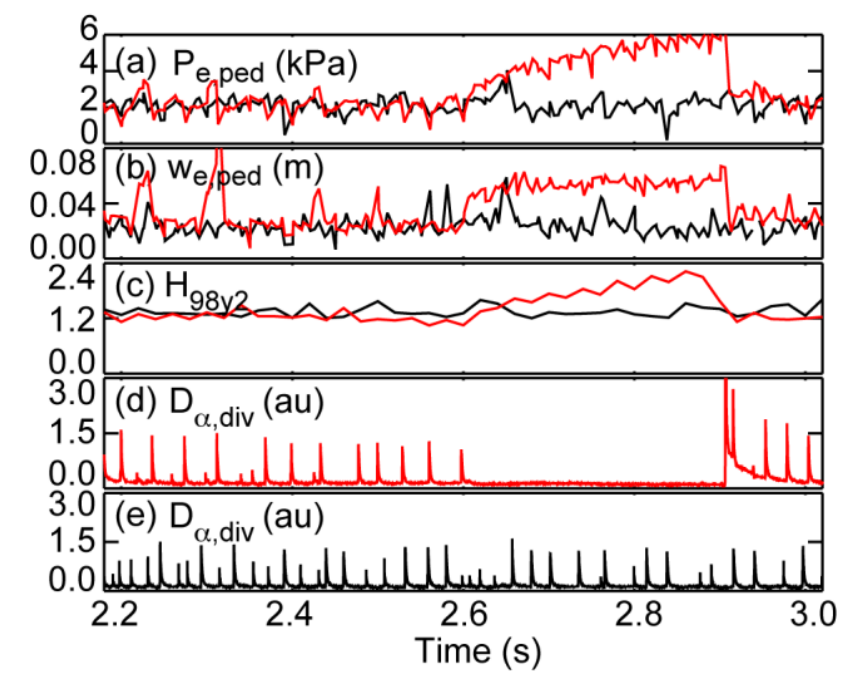

Fig. 5 
Figure 1

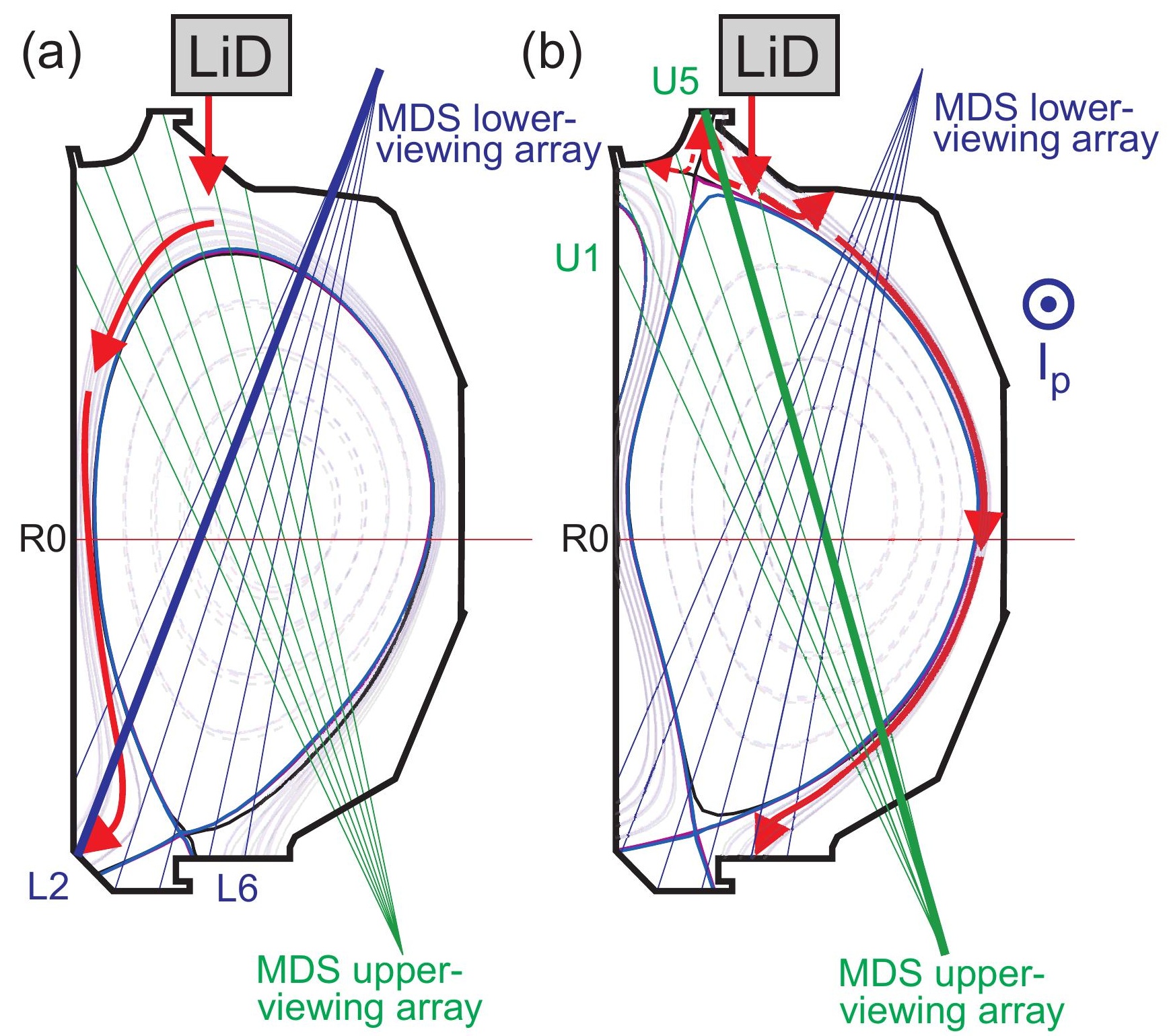


figure 2

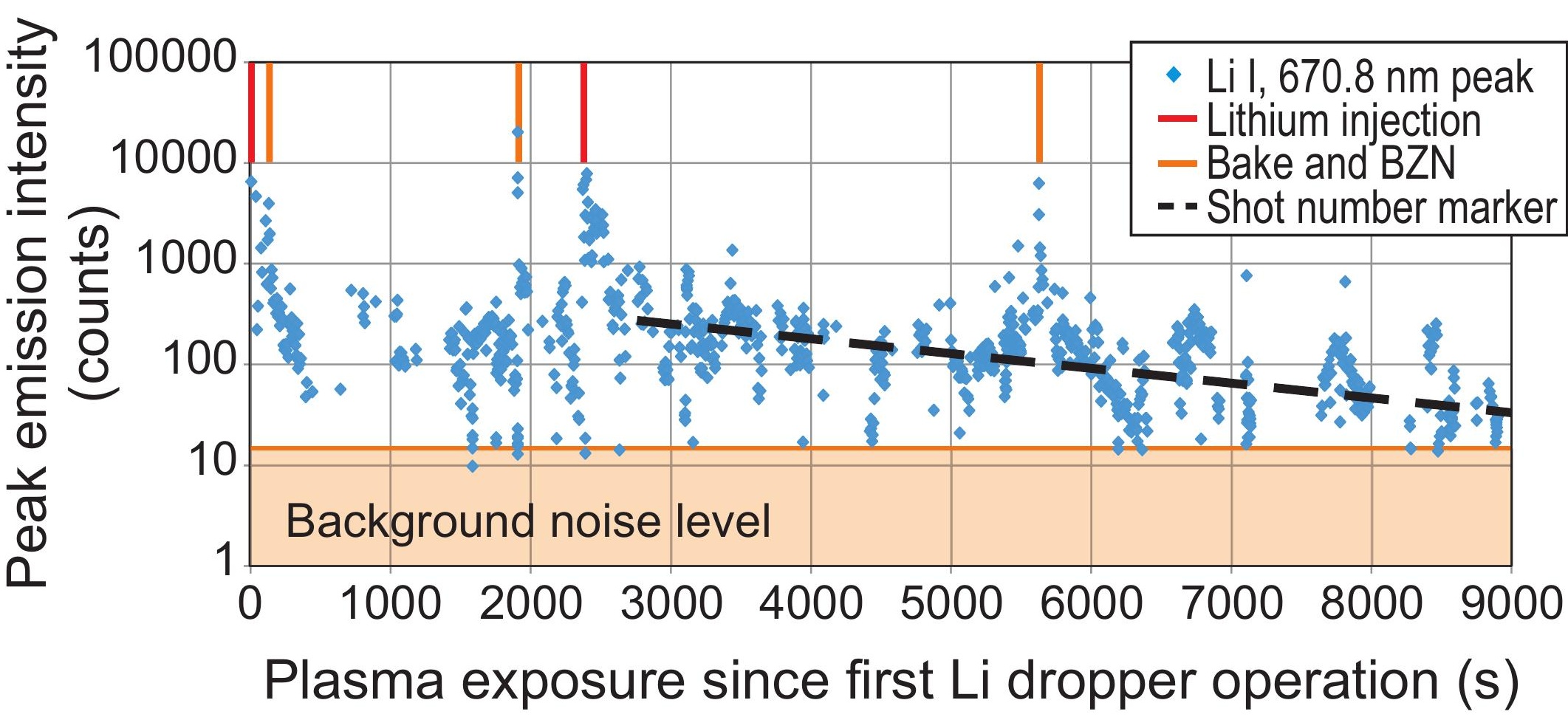


figure 3

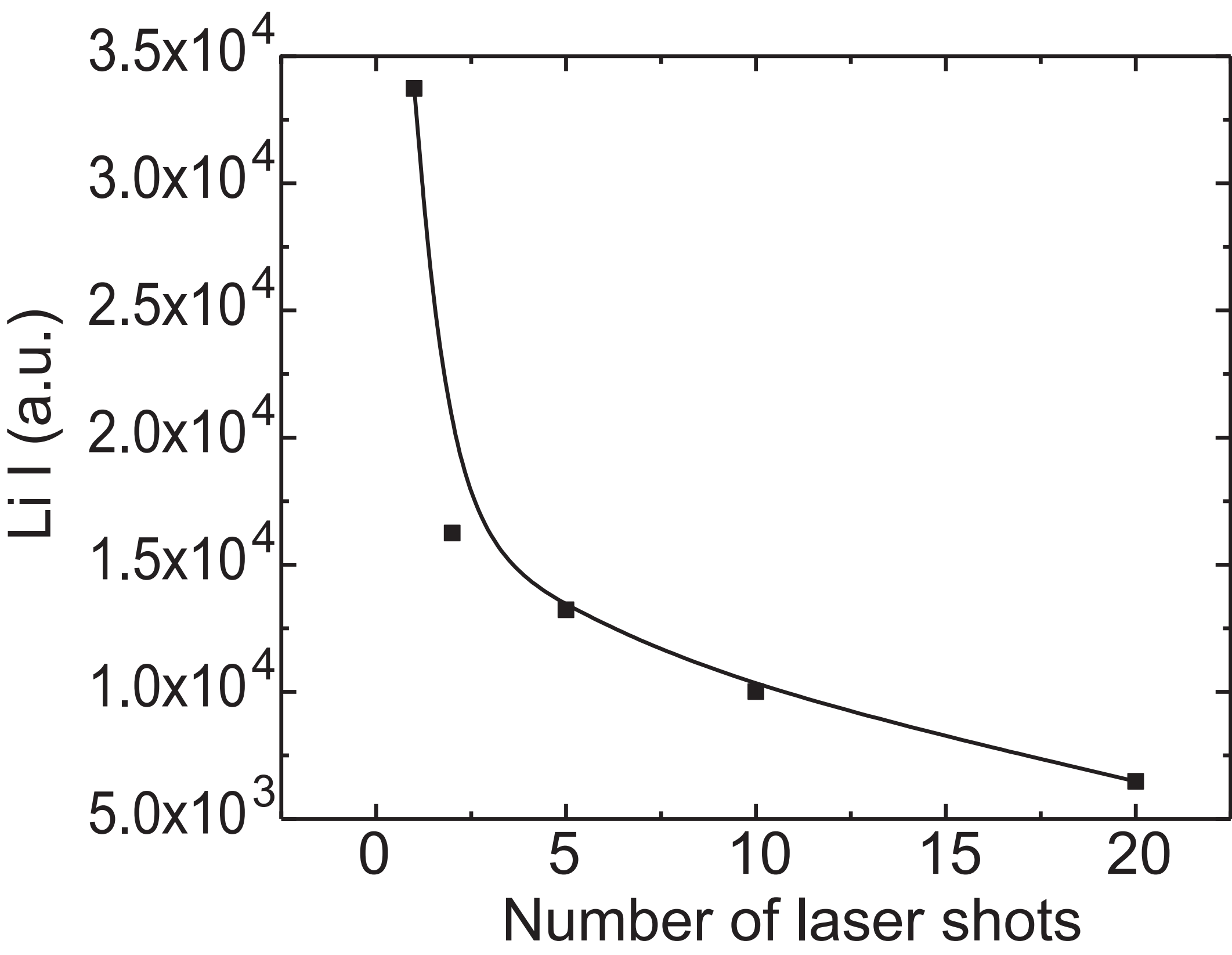


figure 4

\section{$1 \times 10^{16}$}

$D_{\alpha}$

(In. $5 \times 10^{15}$ 152742 Lithium Drop (a)

Div.

au)

$P_{\text {rad }}$

(MW)

$\overline{\mathrm{n}}_{\mathrm{e}}$

$\left(\times 10^{19}\right.$

$\left.m^{-3}\right)$

Li III $2 \times 10^{14}$

(au) $1 \times 10^{14}$

0

15 
figure 5

${ }^{4}$ (a) $P_{\text {eed }}(\mathrm{kPa})$

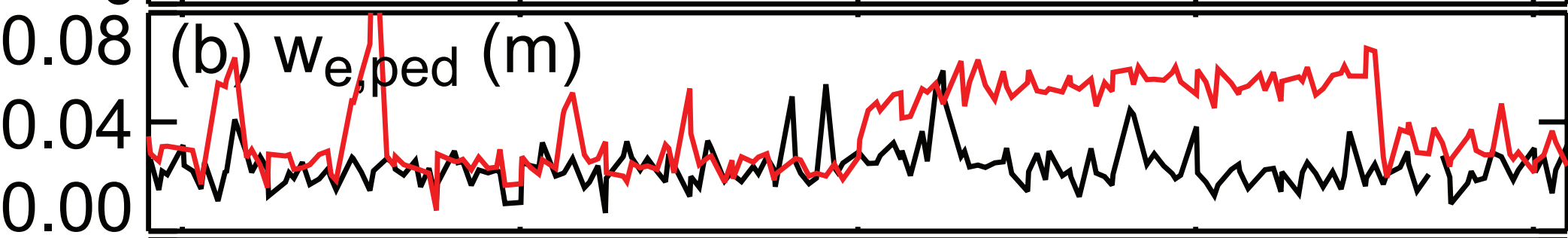

2.4
1.2
0.0

1.5 (d) $\mathrm{D}_{\alpha, \mathrm{div}}(\mathrm{au})$

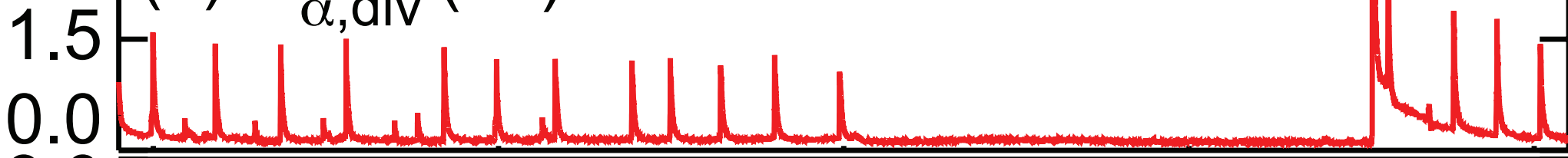

3.0
1.5

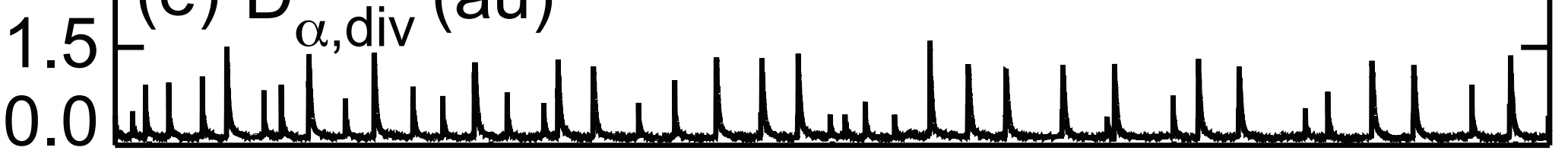

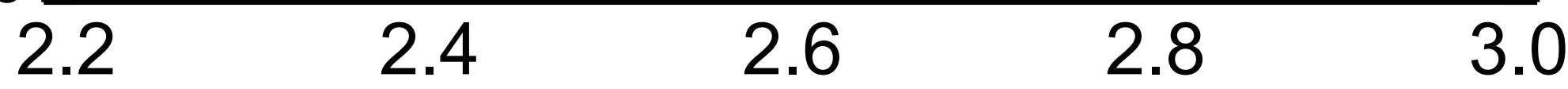

Time (s) 